\title{
Mapping Shallow Sea Depth From Spot-5 Satellite
}

\author{
Luo Kai, Chen Benqing \\ Third Institute of Oceanography, State Oceanic Administrator \\ Daxue Road 178 \\ Xiamen, Fujian, P. R. China \\ luokai@tio.org.cn, 86-592-2195519
}

\begin{abstract}
$^{1}$
Based on the Spot- 5 satellite imagery, this paper uses the wave kinematics bathymetry method to derive shallow sea depth. The Spot-5 satellite has a panchromatic band resolution of $2.5 \mathrm{~m}$ and a width of $60 \mathrm{~km}$, which is suitable for obtaining nearshore gravity wave information. By generating a spectrogram by FFT conversion, the wave number, wavelength, and wave direction information of the sea surface gravity wave can be obtained, and finally the water depth can be obtained. The overall accuracy of the inversion result of water depth in this paper is $18.80 \%$.
\end{abstract}

Key words: WKB, Satellite Imagery, Gravity Wave,FFT

\section{Introduction}

All marine activities, whether economic, military or scientific research activities, such as maritime traffic, marine surveys, marine resource development, maritime defense, etc., require ocean water depth data, so water depth measurement work, especially in offshore waters, is especially important. The emergence of remote sensing technology in the 1960s provided new ideas for offshore bathymetry. In recent years, with the continuous development, remote sensing technology has its unique advantages: wide measurement range, low cost and high efficiency, especially suitable for measurement areas that are difficult to reach by ships. To a certain extent, it can make up for the shortcomings of traditional water depth measurement methods, so it has a very broad application prospect in water depth measurement [1].

In the remote sensing water depth measurement, the data of laser radar and optical image mainly use the absorption relationship between electromagnetic wave and water body to study the inshore water depth inversion [2]. The method has been widely developed [3-6]. Due to the water quality of the nearshore water body, the application of this method is limited to a certain extent[7]. With the rapid development of remote sensing technology, sea surface wave information can be obtained by using high-resolution SAR satellites, optical satellites and other remote sensing data. The nearshore water depth is inferred by using the linear theoretical relationship between shallow sea wave and water depth(WKB) [8-9] .

The WKB is mainly based on the principle that the sea

This work was supported in part by the Scientific Research Foundation of Third Institute of Oceanography, SOA(NO.2014017) and in part by the Promote Cross-Taiwan Strait Union Science and Technology Cooperation Fund(U1405234). surface gravity wave gradually becomes smaller on the nearshore seabed. This principle has been discovered for more than 100 years, but the actual water depth inversion needs to solve two key problems: the first satellite image can provide high-resolution data to extract information about the sea wave, the second computer performance improvement and the implementation of the Fourier transform algorithm. These factors have been resolved with the rapid availability of satellite technology and computer computing [10]. In 1984, Alpers and Hennings (AH) proposed an imaging theoretical model for SAR shallow seawater topography. Then based on the AH model, extensive research on imaging mechanism and imaging theory of SAR shallow seawater topography was carried out. Besides, Ron Abileah et al. Used IKONOS satellite to measure nearshore water depth [11] .and Bradley L extracted wave velocity information from Worldview-2 images, and used the linear relationship between wave velocity and water depth for water depth inversion [12].

In this paper, the wave information of the offshore sea surface gravity wave is extracted from the high-resolution optical satellite image data of the single scene, and the nearshore water depth is inverted by using the linear theoretical relationship between the shallow sea wave velocity, the wave number and the water depth. The advantage of the water depth inversion method based on satellite image extraction wave velocity is that it requires less water quality in the research sea area, and it is more effective to detect the water depth in the sea area with large spatial difference of the seafloor sediment type, and the method overcomes the limitations of multispectral images in turbid waters. Trying to find a simple and effective remote sensing method that can quickly invert the nearshore water depth.

\section{WKB Based On Single Satellite Image}

The basic equation of gravity wave propagation on the sea surface:

$$
C^{2}=\frac{g}{k} \tanh (k h)
$$

$C$ represents the wave velocity of the sea surface gravity wave, $k$ represents the wave number, $h$ represents the water depth, and g represents the gravity acceleration [13].

When gravity waves propagate from the deep sea to the shallow sea, their wave height, wavelength, and wave direction change with the water depth of the propagation zone.

In the medium water depth area there are:

$$
\begin{aligned}
& L=L_{0} \tanh k h \\
& C=C_{0} \tanh k h
\end{aligned}
$$

$L_{0}$ and $C_{0}$ represents the wavelength and wave velocity of the 
deep sea zone, $L$ and $C$ represents the wavelength and wave velocity entering the shallow sea zone.

Assuming that under the condition of no vortex, the wave number $k$ can be obtained:

$$
\frac{\partial(k \sin \theta)}{\partial x}-\frac{\partial(k \cos \theta)}{\partial y}=0
$$

$\theta$ represents the angle of the wave.

Therefore, it is assumed that the contour of certain sea area is straight with the shoreline, and the wave direction changes follow the Snell principle:

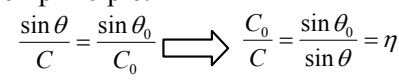

$\theta_{0}$ represents the angle of the wave of the deep sea zone. $\theta$ represents the angle of the wave entering the shallow sea zone.

The satellite image $I(x, y)$ contains the specific coordinate position of the sea wave at a certain moment. After Fourier transform, the satellite image can get the frequency spectrum $S_{t}\left(k_{x}, k_{y}\right)$ of the sea surface at a certain time. Then, the wave number and wave direction can be obtained by frequency spectrum:

$$
\begin{aligned}
& \frac{2 \pi}{\lambda}=k=\sqrt{k_{x}^{2}+k_{y}^{2}} \\
& \theta=\tan ^{-1}\left(k_{x} / k_{y}\right)
\end{aligned}
$$

$k_{x}, k_{y}$ is the value of the peak in the $\mathrm{X}$ and $\mathrm{Y}$ directions in the spectrum, $\theta$ is the angle between the wave propagation direction and the $\mathrm{Y}$ axis.

Based on the above theory, the inversion formula of remote sensing water depth can be obtained as follows:

$$
h=\frac{\operatorname{atan} \mathrm{h}(\eta)}{k}
$$

Where $k$ and $\theta$ have been obtained from the converted spectrogram.

In order to meet the Snell principle, this paper assumes that the isobath of the study area is relatively straight with the shoreline, which restricts some sea areas with irregular water depths for water depth inversion by this method.

\subsection{Research area and data}

\section{Case study}

This paper selects the study of water depth inversion in Da Cheng Bay, southern Fujian Province. The area is located in the Tropic of Cancer and belongs to the southern subtropical maritime monsoon climate. The annual average temperature is $20.8^{\circ} \mathrm{C}$, the lowest temperature in February is $3.8^{\circ} \mathrm{C}$, and the highest temperature in July-August is $36.6{ }^{\circ} \mathrm{C}$. The area has a high wind speed and an annual average wind speed of $6.8 \mathrm{~m} / \mathrm{s}$. The average monthly wind speed reached $8.8 \mathrm{~m} / \mathrm{s}$. The tidal type is an irregular half-day tide type with an average tidal range of $230 \mathrm{~cm}$ and a maximum tidal range of $414 \mathrm{~cm}$.

A total of four satellite images were obtained, namely: WorldView-2, QuickBird, Spot-5, and Landsat-8. The general high-resolution satellites include the panchromatic band and the multi-spectral band, wherein the resolution of the panchromatic band is significantly higher than that of the multi-spectral band, and more abundant wave information can be obtained, which is more suitable for extracting wave information. There are more spectral information in the multi-spectral band, and the near-infrared band and the red band have less radiation relative to the atmospheric path, which can improve the contrast of the sea surface wave. Considering these factors, this paper uses the panchromatic band for water depth inversion.

\subsection{Water depth inversion}

From the land to the sea area, the satellite image $s$ is divided into some cell images by frames, each of which has a size of $1280 \mathrm{~m} * 1280 \mathrm{~m}$ as shown in Fig. 1. The remote sensing images are sequentially splited according to these frames, and each segmented cell image generates frequency spectrum through Fourier transform one by one.

Select the frequency spectrum of the same area with better sea surface waveform for comparison. From Fig. 2, it can be seen that the spectrum of the LandSat- 8 satellite image cannot read the valid $k_{x}$ and $k_{y}$ values. The LandSat- 8 satellite has a resolution of $15 \mathrm{~m}$. Compared with other satellites, the resolution of the satellite is relatively low, and the information of the waves in the image is relatively poor, which is not suitable for water depth inversion research. The $k_{x}$ and $k_{y}$ values in the spectrograms of the Spot-5 and Worldview- 2 satellites in the other three satellites are clearer, and considering that the width of the Spot-5 satellite is significantly larger than the other two satellites, this paper mainly select Spot- 5 satellite imagery for water depth inversion.

From the frequency spectrum obtained by FFT conversion, the gravity wave number and wave propagation angle of the cell image are calculated. The frame of $\theta_{0}$ is located at a deeper depth and farther away from the shore, where the water depth is deeper and the waves are less affected by the seabed topography. This is the reason why this paper chooses the larger width satellite. Finally, the water depth of each cell image can be retrieved by comparing other cell image one by one with the deep sea cell image.

In the Spot-5 inversion results, the wave number, wave direction, and wavelength value of the inversion of 20 cells in one column are selected from the shoreline to the sea area, as shown in TABLE 1 . The variation trend of the gravity wave wavelength of the inversion can be seen in the table: the farther from the shore, the longer the wavelength, the smaller the wave number, and the trend is basically consistent with the trend of the wavelength of the nearshore gravity wave with the water depth.

The water depth of nautical chart is used to verify the water depth inversion results. Since the water depth of our inversion is the instantaneous water depth value of the satellite image capturing time, the real depth is obtained by the inversion water depth subtracting the height of the tide. Fig. 3 is the result of inversion water depth of WKB.

\subsection{Analysis of results}

This paper selects 100 verification points. The water depth verification points are evenly distributed in the inversion area to ensure that the different depth inversion results are compared The total average inversion accuracy of the study area was $18.80 \%$. Accuracy statistics analysis of inversion results of different depths of water depth: 13 verification points in the depth range of $3 \sim 10 \mathrm{~m}$, the average percentage error of inversion is $20.95 \%$; 65 water depth verification points in the range of $10 \sim 20 \mathrm{~m}$ water depth, the average percentage error of inversion is $13.80 \%$; 22 verification points in the depth range of $20 \sim 30 \mathrm{~m}$, the average percentage error of the inversion is $36.25 \%$. 


\section{Recent Developments on Information and Communication Technology (ICT) Engineering- Meen, Yang \& Zhao ISBN: 978-981-14-2136-5}

Fig. 4 shows the absolute error distribution of water depth at all verification points. It can be seen that the verification points with absolute error greater than $4 \mathrm{~m}$ mainly distributes in the sea where the water is deeper than $20 \mathrm{~m}$.

Referring to the nautical chart of the study area, it can be seen that the sea area below $20 \mathrm{~m}$ is located outside of the bay. The sea area is greatly affected by wind and has more spoondrift, so the overall inversion result error is poor. The satellite imagery is taken on January 4, which is just in winter. The wind is strong in this season. The wave waveforms outside the Bay are affected by wind obviously, and the accuracy of inversion results are very poor. When comparing images in the bay and images outside the bay, it can be seen that there are obvious spoondrift in the image of the sea outside the bay, but the waves in the bay are clear and there are not obvious spoondrift inside the bay.

There are two reefs in the bay (the Lao Niu reef and the Qi Xin reef). Around those reefs the water depth changes very obviously, so underwater topography around the reef has a greater influence on the sea wave form, which leads to a large error in the inversion water depth. As shown in Fig. 5, the waveform change near the Lao Niu reef is very obvious. It is difficult to read the exact wave number in the frequency spectrum after FFT, and the error is also obvious around the sea area.

\section{Conclusions and Perspective}

WKB based on single satellite imagery requires satellite imagery to meet certain horizontal resolution in order to extract wave number of nearshore sea surface gravity waves and the width of satellite imagery should be as large as possible. In this paper, the horizontal resolution and width of four medium- and high-resolution satellites (WorldView-2, QuickBird-2, Spot-5, LandSat-8) are compared, and the Spot-5 satellite image is selected for water depth inversion.

WKB mainly extracts the spectral information of the gravity waves on the nearshore sea surface, so the waveform of satellite image must meet certain height. If the sea wave height is too small, it is difficult to obtain the change information of the sea wave from the image. If the wave height of sea surface is too large, there will be too much interference information in the converted frequency spectrum. In this paper, the comparison of wave images inside and outside the bay shows that the large wind waves have more obvious impact on the final results. It is also possible to perform filtering and enhancement processing on image data having large wind wave, and the result precision can be improved to a certain extent. Since the quality of satellite remote sensing images is affected by many factors such as atmospheric conditions, the weather in the required area can be queried through relevant meteorological information when satellite data is selected. Under normal circumstances, the sea surface gravity wave height is $0.5-1.5 \mathrm{~m}$, and the sea surface wind speed is $5-15 \mathrm{kts}$.

In this paper, the formula derivation process assumes that the isobath of the study area is relatively parallel to the shoreline. It can be seen from the inversion results that the sea area with obvious changes in the seabed depth are inferior in accuracy. Especially in sea area with reefs, which have obvious influence on the sea surface waveform, the interference in image cannot be removed by means of image enhancement and other techniques.
The measurement results in this paper are difficult to meet the requirements of the International Hydrographic Standards, but the water depth measurement is faster and the cost is lower. Especially for some remote sea areas that are difficult to reach, this method can provide useful results.

\section{References}

[1] Ye Ming, Li Rendong, Xu Guopeng. Multispectral Remote Sensing Methods And Research Progress [J]. World Sci-Tech R \& D.2007,29(2):76-79.

[2] Gutnther G C, Cunningham A G,LaRocque P E,et al.Meeting the Accuracy Challlenge in Airborne Lidar Bathymetry[C]//The 20th EARSeL Symposioum:Workshop on Lidar Remote Sensing of Land and Sea.Dresden,Germany,European Association of Remote Sensing Laboratories, 200,6:16-17.

[3] Wang Yanjiao, Dong Wenjie, Zhang Peiqun,et al. Research Progress of Visible Light Remote Sensing Method for Water Depth [J]. Marine Science Bulletin,2007,26(5):92-101.

[4] John, M P, Robert, E S. Water depth mapping from passive remote sensing data under a generalized ratio assumption [J]. Applied Optics, 1983, 22(8): 1134-1135.

[5] Lyzenga, D R. Passive remote Sensing techniques for mapping water depth and bottom features [J]. Applied Optics, 1978, 17(3): 379-383.

[6] XiaoYongmao, Li Bingqiu. Study On Remote Sensing of Water Depth in Coral Reef Waters [J]. Remote sensing technology and Application, 1995,5(2):25-27.

[7] Fan K G, Huang W G, Fu B, et al. SAR shallow water bathymetry surveys: a case study in Taiwan Shoal[J]. Chinese Journal of Geophysics, 2012, 55(1):310-316.

[8] R. Almar, R. Cienfuegos, et al. Nearshore bathymetric inversion from video using a fully non-linear Boussinesq wave model[J].Journal of Coastal Research.2011,64:20-24.

[9] Marcello de Michcle, Sebastien Leprince, Jerome Thiebot,et al.Direct Measurement of ocean waves velocity field from a single SPOT-5 dataset[J].Remote Sensing of Environment.2012,119:266-271.

[10] Abileah R. Mapping shallow water depth from satellite[C]// 2006.

[11] Abileah R. Mapping Ocean Currents With IKONOS[C]// Oceans. IEEE, 2007:1-5.

[12] Bradley L McCarthy. Coastal bathymetry using 8-color multispectral satellite observation of wave motion. [D]. California: Monterey California Naval Postgraduate School, 2010.

[13] Feng ShiJie. Introduction to marine science [M]. higher education press, 1999.202 
Recent Developments on Information and

Communication Technology (ICT) Engineering-Meen, Yang \& Zhao

ISBN: 978-981-14-2136-5

TABLE 1 Wave number, wave direction, and wavelength inversion result of a line

\begin{tabular}{|c|c|c|c|}
\hline NO. & Wave number & $\begin{array}{c}\text { the angle of the } \\
\text { wave } \\
\left({ }^{\circ}\right)\end{array}$ & $\begin{array}{c}\text { Wave } \\
\text { length(m) }\end{array}$ \\
\hline 1 & -- & -- & -- \\
\hline 2 & 0.076675 & 0.6948 & 81.90 \\
\hline 3 & 0.083593 & 0.7023 & 75.13 \\
\hline 4 & 0.085868 & 1.0304 & 73.14 \\
\hline 5 & 0.072970 & 1.2278 & 86.06 \\
\hline 6 & 0.062865 & 0.8961 & 99.90 \\
\hline 7 & 0.069770 & 0.8851 & 90.01 \\
\hline 8 & 0.064005 & 0.5667 & 98.12 \\
\hline 9 & 0.055536 & 0.7854 & 113.08 \\
\hline 10 & 0.053096 & 0.9828 & 118.28 \\
\hline 11 & 0.055969 & 0.9098 & 112.21 \\
\hline 12 & 0.059920 & 0.6107 & 104.81 \\
\hline 13 & 0.066043 & 0.7328 & 95.09 \\
\hline 14 & 0.059110 & 0.7266 & 106.24 \\
\hline 15 & 0.046307 & 1.0123 & 135.62 \\
\hline 16 & 0.055969 & 0.9098 & 112.21 \\
\hline 17 & 0.046571 & 1.2490 & 134.85 \\
\hline 18 & 0.057246 & 1.0304 & 109.70 \\
\hline 19 & 0.041942 & 1.2119 & 149.73 \\
\hline 20 & 0.057246 & 1.0304 & 109.70 \\
\hline & & & \\
\hline
\end{tabular}

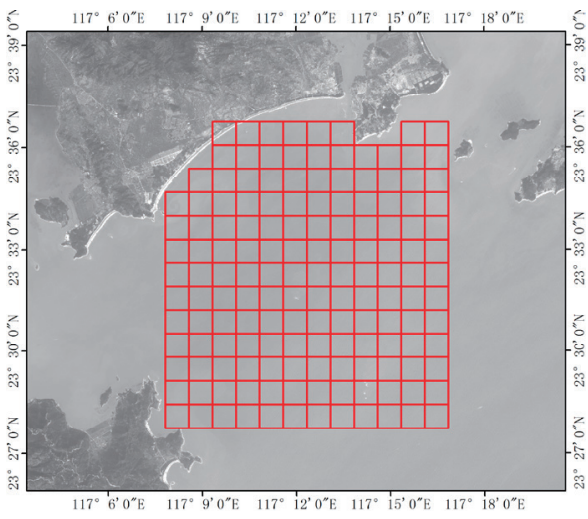

Fig. 1 Sub-frame division map of the inversion area
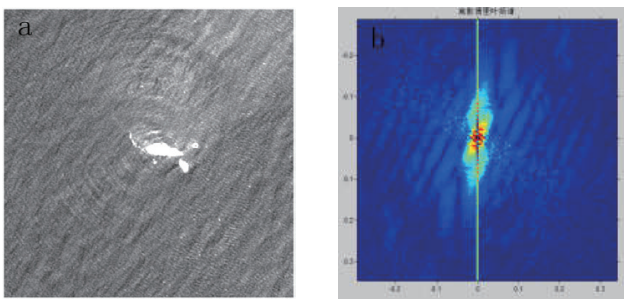

Fig. 5 Satellite image and frequency spectrum of the Lao Niu reef(a. satellite image, b. frequency spectrum)
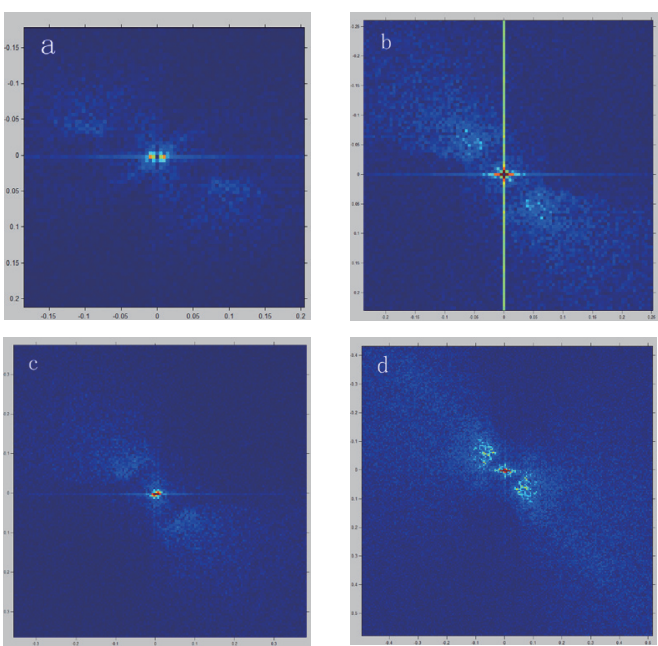

Fig. 2 Comparison of four satellite frequency spectra(a. LandSat-8, b. Spot-5, c. QuickBird, d. WorldView-2)

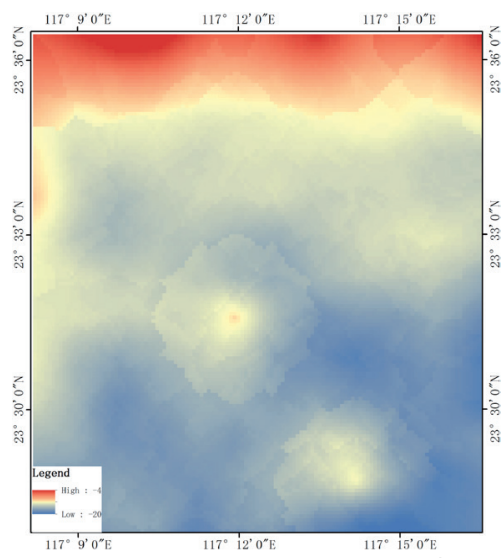

Fig. 3 Water depth inversion results of Da Cheng Bay

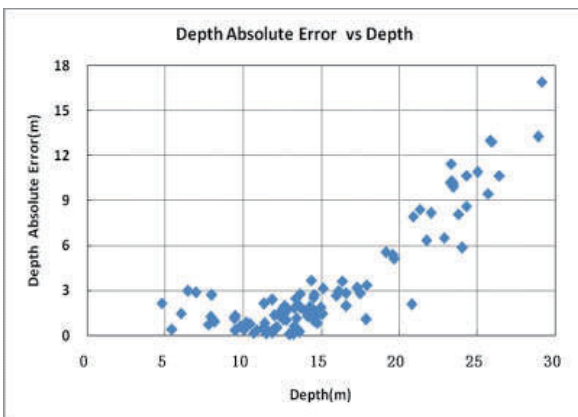

Fig. 4 Absolute error statistics of Da Cheng bay water depth 